\title{
The spice for joint inflammation: anti-inflammatory role of curcumin in treating osteoarthritis
}

\author{
This article was published in the following Dove Press journal: \\ Drug Design, Development and Therapy \\ 20 September 2016 \\ Number of times this article has been viewed
}

\section{Kok-Yong Chin}

Department of Pharmacology, Universiti Kebangsaan Malaysia Medical Centre, Cheras, Malaysia
Correspondence: Kok-Yong Chin Department of Pharmacology, Level I7, Preclinical Building, Universiti Kebangsaan Malaysia Medical Centre, Jalan Yaacob Latif, Bandar Tun Razak, 56000 Cheras, Kuala Lumpur, Malaysia

Tel +60 $39 \mid 459573$

Email chinkokyong@ppukm.ukm.edu.my

\begin{abstract}
Osteoarthritis is a degenerative disease of the joint affecting aging populations worldwide. It has an underlying inflammatory cause, which contributes to the loss of chondrocytes, leading to diminished cartilage layer at the affected joints. Compounds with antiinflammatory properties are potential treatment agents for osteoarthritis. Curcumin derived from Curcuma species is an anti-inflammatory compound as such. This review aims to summarize the antiosteoarthritic effects of curcumin derived from clinical and preclinical studies. Many clinical trials have been conducted to determine the effectiveness of curcumin in osteoarthritic patients. Extracts of Curcuma species, curcuminoids and enhanced curcumin, were used in these studies. Patients with osteoarthritis showed improvement in pain, physical function, and quality of life after taking curcumin. They also reported reduced concomitant usage of analgesics and side effects during treatment. In vitro studies demonstrated that curcumin could prevent the apoptosis of chondrocytes, suppress the release of proteoglycans and metal metalloproteases and expression of cyclooxygenase, prostaglandin E-2, and inflammatory cytokines in chondrocytes. These were achieved by blocking the activation of nuclear factor kappa-light-chain-enhancer of activated B cells (NF- $\mathrm{B}$ ) system in the chondrocytes, by preventing the activation of nuclear factor of kappa light polypeptide gene enhancer in B-cells inhibitor, alpha, phosphorylation, and translocation of the $\mathrm{p} 65$ subunit of NF- $\mathrm{KB}$ complexes into the nucleus. In conclusion, curcumin is a potential candidate for the treatment of osteoarthritis. More well-planned randomized control trials and enhanced curcumin formulation are required to justify the use of curcumin in treating osteoarthritis.
\end{abstract}

Keywords: cartilage, chondrocyte, Curcuma, inflammation, joint, pain

\section{Introduction}

Osteoarthritis is a degenerative disease of the movable joints affecting the elderly population worldwide. It is characterized by localized loss of cartilage, remodeling of adjacent bone, and bony overgrowth. It is the consequence of a maladaptive repair process of the cartilage due to overwhelming trauma. Its presentation is highly variable between individuals, with some common features, such as joint pain, impaired movement, tenderness, crepitus, occasional effusion, and local inflammation. ${ }^{1-3}$ The Osteoarthritis Expert Group of the Global Burden of Disease 2010 Study reported that the prevalence of knee osteoarthritis worldwide was 3.8\% (95\% uncertainty interval [UI]: 3.6\%-4.1\%), and it is higher among females (4.8\%; 95\% UI: 4.4\%-5.2\%) compared to males $(2.8 \%, 95 \%$ UI: $2.6 \%-3.1 \%) .{ }^{4}$ Hip osteoarthritis occurred at lower rate $(0.85 \%$; $95 \%$ UI: $0.75 \%-1.02 \%)$, but it was still more prevalent in females $(0.98 \%$; 95\% UI: $0.82 \%-1.29 \%$ ) compared to males (0.70; 95\% UI: $0.58 \%-0.90 \%) .{ }^{4}$ The Third National Health and Nutrition Examination Survey of the United States revealed that $37.4 \%$ of the elderly population aged 60 years above (females: $42.1 \%$ and males: $31.2 \%$ ) 
suffered from radiographic arthritis, in which $12.1 \%$ were symptomatic. ${ }^{5}$ This translated to one in every ten Americans, or 4.3 million elderly population. ${ }^{5}$ Osteoarthritis is not fatal, but it increases the all-cause mortality rate among the patients (standardized mortality ratio [SMR]: 1.55, 95\% confidence interval [CI]: 1.41-1.70), particularly death related to cardiovascular system (SMR: 1.71, 95\% CI: 1.49-1.99) and dementia (SMR: $1.99,95 \%$ CI: $1.22 \%-3.25 \%) .{ }^{6}$ Despite the higher prevalence in females, mortality rate in osteoarthritic males is higher (SMR: 1.59, 95\% CI: 1.30-1.96). ${ }^{6}$ Osteoarthritis ranks eleventh in terms of years of life lived with disease, above asthma, ischemic heart disease, and Alzheimer's disease. ${ }^{7}$ It incurs significant health care and economic burden to the patients. A study by Ruiz et al reported that the cost for total knee replacement for end-stage osteoarthritic patients was approximately 20,635 USD, and the societal cost was around 39,565 USD due to loss of productivity, employment, earning, and missed working days. ${ }^{8}$ The net societal saving for knee arthroplasties procedure for the year 2009 in the US alone was 12 billion USD. ${ }^{8}$

Osteoarthritis is an inflammatory disease, although the extent of inflammation is lower compared to rheumatoid arthritis. Kraus et al proposed that altered inflammatory state is the underlying cause of osteoarthritis, and mechanical stress is the inducer. ${ }^{1}$ Patients with arthroscopic manifestation of early osteoarthritis and knee pain but normal radiographs $(n=10$, mean age: 63.4 years) exhibited significantly higher immunohistological measures of inflammation, such as tumor necrosis factor alpha (TNF $\alpha)$, interleukin 1 beta (IL-1 $\beta$ ), nuclear factor kappa-light-chain-enhancer of activated $B$ cells $(\mathrm{NF}-\kappa \mathrm{B})$, and cyclooxygenase 2 (COX-2) in synovial tissue compared to late osteoarthritic patients who required arthroplasty ( $n=15$, mean age: 74.4 years).${ }^{9}$ Another study by Sohn et al showed that 108 proteins were differentially expressed in the synovial fluid of osteoarthritic patients compared to normal patients. These proteins included plasma proteins, serine protease inhibitors, cartilage turnover proteins, and inflammatory and immunity proteins. Some of the plasma proteins, such as Gc-globulin, alpha 1-, and alpha 2-microglobulin, could promote the secretion of inflammatory cytokines by macrophages through interaction with toll-like receptor $4 .{ }^{10}$ Local damage to the joint due to trauma or overuse can cause pathological inflammation, which serves as the initiator of osteoarthritis. Inflammation stimulates the release of damage associated molecular patterns, such as fibronectin and hyaluronan, from the breakdown of cartilage extracellular matrix. These damage associated molecular patterns further enhance the release of inflammatory cytokines TNF $\alpha$ and
IL-1 $\beta$, and matrix metalloproteinases (MMP)-1 and -3 to promote chondrolysis and breakdown of extracellular matrix, thus forming a vicious cycle. The inflammation is further promoted by alarmins released by stressed and dying chondrocytes and plasma proteins that enter the joint space due to vascular leakage resulting from local damage. ${ }^{11}$ Oxidative stress is implicated in the inflammatory process of osteoarthritis. Under the stimulation of TNF $\alpha$ and IL-1 $\beta$, adenosine triphosphate production of chondrocytes is reduced due to the inhibition of complex I in mitochondrial respiratory chain, thereby reducing the mitochondrial membrane potential. At the same time, nitric oxide, free oxygen species, and superoxide anions released by macrophages and mitochondrial dysfunction can result in the death of chondrocytes. ${ }^{12}$

Pharmacological treatments currently available for osteoarthritis include paracetamol (acetaminophen) as the first-line treatment, oral and topical nonsteroidal anti-inflammatory agents (NSAIDs) as the second-line treatment, tramadol and intra-articular corticosteroid injections. Opioids, duloxetine, and intra-articular hyaluronate injections are recommended when these initial treatments fail. Nonpharmacological modalities included biomechanical interventions, land-based and water-based exercise, strength training, and weight management. ${ }^{13-15}$ The efficacy of glucosamine sulfate, chondroitin sulphate, and avocado soybean unsaponifiables remains uncertain. ${ }^{16}$ Most of these treatments relieve the symptoms of osteoarthritis, but they do not modify the underlying cause of the disease, which is chronic inflammation. While nonselective NSAIDs can suppress inflammation, they are known to be associated with gastrointestinal toxicity (relative risk [RR]: 5.36, 95\% CI: $1.79-16.10) .{ }^{17}$ Selective NSAIDs such as celecoxib (RR: 1.12, 95\% CI: 1.00-1.24), rofecoxib (RR: 1.34, 95\% CI: 1.22-1.48), and etoricoxib (RR: 1.97, 95\% CI: 1.35-2.89), are associated with acute myocardial infarction. ${ }^{18}$ Total knee replacement is recommended for end-stage osteoarthritic patients but it has been associated with risk of deep infection and deep vein thrombosis. ${ }^{19}$ The quest for an effective treatment regime with fewer side effects is on-going.

One of the potential antiosteoarthritic agents under limelight is curcumin. Curcumin is derived from turmeric, a popular spice used in India, South Asia, and Japan, which is the grounded root and rhizome of the plant Curcuma longa. In traditional Indian medicine, turmeric is used to treat biliary digestive disorder, wounds, and rheumatic diseases. Besides other macro- and micronutrients, the extract of turmeric contains $77 \%$ curcumin, $17 \%$ demethoxycurcumin, and $3 \%$ bisdemethoxycurcumin, collectively named curcuminoids, 
<smiles>COc1cc(/C=C/C(=O)CC(=O)/C=C/c2ccc(O)c(OC)c2)ccc1O</smiles>

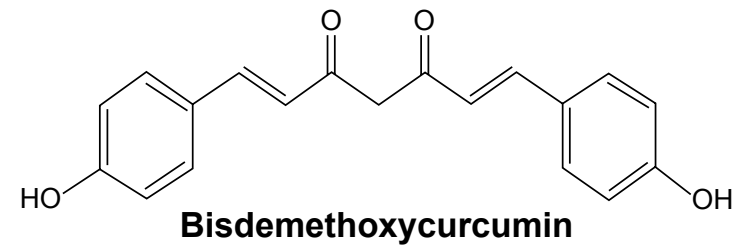<smiles>COc1cc(/C=C/C(=O)CC(=O)/C=C/c2ccc(O)cc2)ccc1O</smiles>

Figure I Chemical structure of curcumin, demethoxycurcumin, and bisdemethoxycurcumin.

which render turmeric its distinct yellowish hue (Figure 1)..$^{20,21}$ Curcumin possesses both anti-inflammatory and antioxidative activities. Curcumin has been shown to suppress the activation of IKB (inhibitor of nuclear factor kappa-lightchain-enhancer of activated B cells [NF-KB]) kinase (IKK) and the phosphorylation of $\mathrm{AKT}$ and the association between the two signaling molecules induced by IL-1 $\beta .{ }^{22}$ In turn, this results in the inhibition of phosphorylation and degradation of nuclear factor of kappa light polypeptide gene enhancer in B-cells inhibitor, alpha (IKB $\alpha)$, the endogenous blocker of NF- $\kappa B .{ }^{22}$ Therefore, curcumin is able to inhibit translocation of NF- $\mathrm{KB}$ into the nucleus, thus preventing the inflammation response of the cells. ${ }^{22}$ Curcumin also prevents the decrease of Bcl-2 and Bcl-XL expressions and the increase of Bax and caspase- 3 expressions stimulated by IL-1 $13.22,23$ Decrease of $\mathrm{Bcl}-2 / \mathrm{Bax}$ and $\mathrm{Bcl}-\mathrm{XL} / \mathrm{Bad}$ ratio is associated with the loss of mitochondrial membrane potential. ${ }^{23}$ It also suppresses the phosphorylation of p38 and c-Jun N-terminal kinases (JNK), and increase expression of ERK and AKT serving as prosurvival signal, thereby inhibiting apoptosis. ${ }^{23}$ On the other hand, curcumin increases the phosphorylation and DNA binding activity of nuclear factor erythroid 2-related factor 2 to antioxidant response element, ${ }^{24,25}$ besides acting as free radical scavenger itself. ${ }^{26}$ These translated to reduced inflammation and oxidative stress in experimental animals and humans supplemented with curcumin. ${ }^{27-31}$ This preliminary evidence justifies the potential of curcumin as an antiosteoarthritic agent.
The objective of this review is to summarize the clinical and preclinical evidence of curcumin as an antiarthritic agent and the putative mechanisms involved for its actions.

\section{Literature search}

Literature search was performed from May 1, 2016 to May 31, 2016, with keywords "curcumin OR curcuminoid OR Curcuma” AND “osteoarthritis” using Scopus, Pubmed, and Web of Science. Only original research articles written in English were selected. Both clinical and preclinical studies were included. Full texts of the relevant articles were extracted after being screened for titles and abstracts.

\section{Clinical studies}

A total of 15 relevant clinical studies on the effects of curcumin supplementation on osteoarthritic patients were retrieved. Among the 13 randomized controlled trials, five compared the effectiveness of curcumin with a placebo; ${ }^{32-36}$ four with NSAIDs (ibuprofen, ${ }^{37,38}$ celecoxib, ${ }^{39}$ and diclofenac sodium $^{40}$ ); two with the best available treatment decided by the attending physicians, ${ }^{41,42}$ one with chondroitin, ${ }^{43}$ and one compared currently with glucosamine and placebo. ${ }^{44}$ The remaining two studies were time series studies without a control group. ${ }^{45,46}$ The treatments assigned to the patients included crude extracts of Curcuma domestica, ${ }^{38}$ Curcuma longa alone, ${ }^{44}$ or with Boswellia serrate, ${ }^{32,39}$ curcuminoids alone, ${ }^{40}$ or with piperine, ${ }^{34-36}$ and curcuminoids improved in bioavailability. ${ }^{33,41-43,45}$ Dosage of curcumin used in these studies ranged from $180 \mathrm{mg}^{33}$ to $2,000 \mathrm{mg}^{38}$ daily. The treatment period ranged from 4 weeks ${ }^{40}$ to 8 months ${ }^{41}$ Endpoints assessment included Western Ontario and McMaster Universities Arthritis Index (WOMAC), Karnofsky Index, visual analogue scale, mobility test, and various physical assessments. Four studies ${ }^{32,36,41,46}$ determined the circulating inflammatory cytokine levels and two studies ${ }^{32,34}$ determined markers of oxidative stress in patients. The following discussion will be based on the formulation of the curcumin used in these studies.

A combination extract of C. longa and boswellia (botanical resin used in Ayurvedic medicine) was examined in the earliest osteoarthritis study. In a double-blinded randomized controlled trial by Badria et al, 30 outpatients (age not disclosed) with osteoarthritis were prescribed a combination of extracts of C. longa and Boswellia carteri (percentage of curcumin in the combination unknown) for 3 months. The treatment group showed significant improvements in the pain-free walking time, degree of pain before and after passive and active movement tenderness, and grade of knee 
effusion compared to placebo. This was attributed to the reduction in inflammation indicated by $\mathrm{CD}^{+}$and $\mathrm{CD} 45 \mathrm{RO}^{+}$ T-cells and serum Fas ligand, as well as oxidative stress indicated by serum nitrite/nitrate and superoxide dismutase levels in the patients. ${ }^{32}$ Another study by Kizhakkedath compared the effects of the combination of $700 \mathrm{mg} \mathrm{C}$. longa and $300 \mathrm{mg}$ $B$. serrate extract ( $\mathrm{n}=14$; mean age: 49.70 [8.20] years) and $200 \mathrm{mg}$ celecoxib ( $\mathrm{n}=14$; mean age: 47.20 [9.70] years) for 12 weeks. Categorical analysis revealed that the number of patients suffering from moderate-to-severe joint pain and joint line tenderness was reduced in the treatment group compared to the placebo group. However, the number of patients who could walk $<500 \mathrm{~m}$ and suffering from crepitus was not different between the two groups. ${ }^{39}$ Both studies recruited a small number of subjects and the duration of treatment was rather short. Due to the nature of the combination, individual effects of curcumin on osteoarthritis patients could not be assessed. The study by Kulkarni et al examining a herbomineral formulation containing $C$. longa extract on osteoarthritic patients would not be discussed here due to the low content of Curcuma extract in the formulation (10\% weight/weight). ${ }^{47}$

C. longa extract alone was proven to be an effective treatment for osteoarthritis. Madhu et al treated patients with radiographic osteoarthritis grade $2-3$ based on the Kellgren and Lawrence scale with 1,000 mg C. longa extract (n=29), $1,500 \mathrm{mg}$ glucosamine $(\mathrm{n}=28)$, the combination of both $(n=24)$, and placebo $(n=29)$ for 42 days (mean age: 57.09 [standard deviation (SD): 9.41] years). C. longa extract alone was more effective in improving all WOMAC subscales and total score, clinician global impression of change, joint tenderness, crepitation, effusion, and limitation to movement than the combination and placebo group. The number of patients needing paracetamol and cases of adverse reaction was also the lowest in group taking $C$. longa extract alone. ${ }^{44}$ This study is single-blinded and falls short in the number of the subjects and treatment duration. In other studies using C. domestica extract, Kertia et al demonstrated that daily supplementation of $120 \mathrm{mg}$ of curcuminoids extracted from C. domestica for 4 weeks $(\mathrm{n}=34)$ was as effective as $75 \mathrm{mg}$ of diclofenac sodium $(n=39)$ in suppressing the synthesis of COX-2 in monocytes in the synovial fluid of patients (mean age: 64.05 [SD: 8.83] years) suffering from mildto-moderate osteoarthritis diagnosed using the criteria of American College of Rheumatology. ${ }^{40}$ However, it was unclear whether the patients benefited from such a low dose of curcumin supplementation in terms of changes in clinical outcomes and pain relief. Kuptniratsaikul et al compared the efficacy of $C$. domestica extract (2,000 $\mathrm{mg}$ per day) and ibuprofen ( $800 \mathrm{mg}$ per day) for 6 weeks in patients (mean age: 61.4 [SD: 8.7 ] years) with radiographic osteoarthritis. They observed no significant changes in severity of pain and time needed for walking and climbing stairs between the two groups. The number of patients suffering from adverse reactions and their level of satisfaction were also similar between the two groups. ${ }^{38}$ However, it should be noted that the dose for ibuprofen in this study was suboptimal, thus the comparison might not be fair. To counter the problems of small sample size and dose of ibuprofen, a multicenter study was conducted. A total of 185 osteoarthritic patients (mean age: 60.3 [SD: 6.8] years) received 1,500 mg extract of C. domestica, and 160 patients received 1,200 mg ibuprofen per day for 4 weeks. Both groups exhibited similar improvements in WOMAC subscales and total score at the end of the treatment period. The occurrence of adverse effects, such as dyspepsia, abdominal pain, nausea, loose stool, and edema, patients' global assessment, and satisfaction were not different between the two groups. ${ }^{37}$ This is the largest curcumin study on osteoarthritis recorded to date and it benefits from having a large sample size, proper blinding and randomization, despite a short duration of treatment.

The bioavailability of curcumin is very low. An animal study in rats revealed that a single oral dosage of curcumin at $500 \mathrm{mg} / \mathrm{kg}$ resulted in a maximum serum concentration of $0.06 \mu \mathrm{g} / \mathrm{mL}$ (SD: 0.01 ) at 41.7 minutes (SD: 5.4), equivalent to an oral bioavailability of $1 \%{ }^{48}$ There are various attempts to improve the bioavailability of curcumin, including delivering curcumin in liposomes or solid lipid nanoparticles, polymeric micelles, or nanoparticles. ${ }^{49}$ The peak plasma level and concentration time curve of curcumin in rats receiving the compound formulated with phosphatidylcholine (Meriva) orally were shown to be five times higher than rats receiving unformulated curcumin. ${ }^{50}$ The efficacy of Meriva has been tested in patients with osteoarthritis. ${ }^{41-43}$ In a pilot study, patients with radiographic bilateral osteoarthritis suffering from mild-to-moderate pain were randomized into the treatment group ( $n=50$; mean age: 44.4 [SD: 7.2] years) receiving 1,000 mg Meriva tablets containing $200 \mathrm{mg}$ curcuminoids and the best available treatment given by the physicians for 3 months and the control group $(n=50$; mean age: 45.3 [SD: 8.6] years) receiving only the best available treatment. Significant improvements in WOMAC scores across all subscales (pain, stiffness, physical function, social, emotional) and treadmill test were observed in the treatment group but not in the control group. Adverse effects (distal edema, gastrointestinal complications) and management 
costs (pharmacological and nonpharmacological) were significantly reduced in the treatment group compared to the control group. The treatment group also experienced a greater decrease in C-reactive protein value compared to the control group. ${ }^{42}$ Similar improvements were demonstrated in an extended 8-month study using the same treatment, with additional betterments in Karnofsky Performance Scale Index and expanded inflammation panel (serum CD40L, IL-1 $\beta$, IL-6, vascular cell adhesion molecule-1, and erythrocyte sedimentation rate) in the treatment group. ${ }^{41}$ In their latest study, the effectiveness of daily $500 \mathrm{mg}$ Meriva plus $500 \mathrm{mg}$ glucosamine (MG group; $\mathrm{n}=63$ ) was compared to $400 \mathrm{mg}$ chondroitin plus $415 \mathrm{mg}$ glucosamine (CG group; $\mathrm{n}=61$ ) for 4 months (mean age of subjects: 56.4 [SD: 5.2] years). The MG group showed greater improvements in all subscales of WOMAC and Karnofsky Index compared to the CG group. The improvement in treadmill test for the MG group started as early as 1 month after treatment. The rate of concomitant drug use, hospital management cost, and complications (distal edema and gastrointestinal) were significantly lower in the MG group compared to the CG group. ${ }^{43}$ The patients in these studies were relatively young (mean age $<60$ years), hence it is unclear that similar effects will be observed in older subjects with more severe osteoarthritis. The sample size of these studies was limited, but duration was relatively long (up to 8 months). Thus, the long-term beneficial and adverse effects of Meriva could be assessed.

Flexofytol, another optimized curcumin formation with emulsifier polysorbate 80 , has been tested in osteoarthritic patients. In a time series study by Henrotin et al, 22 osteoarthritic patients (mean age: 64.3 [SD: 8.4] years) were given six caps of Flexofytol daily (equivalent to $252 \mathrm{mg}$ curcumin) for 84 days. The treatment significantly reduced circulating markers of collagen degradation, such as Coll2-1, Fib3-1, Fib3-2, Cartilaps, and myeloperoxidase and marker of inflammation, C-reactive protein, in the patients. The global assessment of disease activity assessed using a visual analogue scale was significantly reduced despite a marginal decrease in pain score. ${ }^{46}$ In another large-scale time series study, 820 osteoarthritic patients (mean age: 64.2 [SD: 12.4] years) taking four to six capsules of Flexofytol daily for at least 6 months were surveyed and their physical improvement was assessed by their attending general physicians using a 10-point scoring system. The patients noted a decrease in the severity of pain at all sites and improvements in mobility and quality of life starting at week 6 . The treatment also improved flexibility of the patients at all sites, particularly at hand, and reduced concomitant medication use. Some patients reported the adverse effects of gastrointestinal discomfort. ${ }^{45}$ In these two studies using Flexofytol, the endpoints were not determined using the standardized method of assessments, such as WOMAC and Karnofsky Index, thus hindering comparison with other studies. Apart from that, they are not controlled trials, hence the results are hypothesis-generating at best. Literature search conducted at the time of writing revealed no study on the bioavailability of Flexofytol.

Theracurmin is a curcumin formulation dispensed with colloidal submicron-particles. Pharmacokinetic studies comparing different enhanced curcumin formulations revealed that peak plasma curcumin concentration and area under the concentration-time curve of Theracurmin in capsule or liquid form were significantly higher compared to other formulations in the market. ${ }^{51,52}$ Safety studies also revealed that Theracurmin up to $400 \mathrm{mg}$ for 9 months was safe in cancer patients receiving chemotherapy. ${ }^{53} \mathrm{~A}$ study by Nakagawa et al showed that supplementation of $180 \mathrm{mg}$ of Theracurmin daily in patients with osteoarthritis grade 2 or 3 based on Kellgren and Lawrence scale ( $\mathrm{n}=15$; mean age: 68.7 [SD: 7.0] years) for 8 weeks reduced the severity of pain and rate of concomitant celecoxib use. However, there was no significant change in the disease assessment score determined by Japanese Knee Osteoarthritis Measure between the treatment and the placebo group. ${ }^{33} \mathrm{~A}$ large sample size and a longer duration of treatment are warranted to validate the findings of this study.

Piperine is a natural inhibitor of UDP-glucuronyltransferase in the liver. ${ }^{54}$ Concomitant administration of piperine and curcumin was shown to increase the half-life and bioavailability of curcumin and reduce its clearance. ${ }^{55} \mathrm{In}$ humans, concurrent administration of 2,000 $\mathrm{mg}$ curcumin and $20 \mathrm{mg}$ piperine enhanced the bioavailability of curcumin by $2,000 \% .{ }^{55}$ The effects of concomitant piperine and curcumin administration on osteoarthritis patients have been evaluated. In a randomized double-blinded placebo-controlled trial, patients with mild-to-moderate primary osteoarthritis and pain with active movement were allocated to the treatment group ( $\mathrm{n}=19$; mean age: 57.32 [SD: 8.78] years) receiving $1,500 \mathrm{mg}$ curcuminoids and $15 \mathrm{mg}$ piperine daily for 6 weeks or placebo group ( $\mathrm{n}=21$; mean age: 57.57 [SD: 9.05] years) receiving inert starch. Both groups showed a significant reduction in WOMAC pain stiffness scales after 6 weeks of treatment. Curcuminoids alone caused a significant decrease in WOMAC physical function and global score. The improvements of the treatment group in WOMAC pain index, physical function, and global score were significantly better compared to placebo. Pain measured by Lequesne's 
index and visual analogue scale was significantly lower in the treatment group compared to the control group. ${ }^{35}$ Further analysis revealed that inflammation, indicated by circulating IL-4, IL-6, and TGF- $\beta$ levels, was reduced by the treatment after 6 weeks of treatment. ${ }^{36}$ However, the difference in rate of change was not significant between the treatment and control groups. While this observation did not seem to agree with the physical improvement among the patients, inflammation in osteoarthritis is localized at the joint rather than systemic. Thus, circulating inflammatory markers might not reflect the local inflammatory status of the joint. With respect to the oxidative level, the treatment group showed an increased superoxide dismutase and glutathione level and a reduced malondialdehyde level (a product of lipid peroxidation) compared to placebo. ${ }^{34}$ The assessment of these biochemical markers of oxidative status was conducted using simple spectrophotometry, which could be improved using more sensitive and reliable methods. Overall, these studies involved only a small number of subjects and a short duration of time.

Overall, the extensive clinical trials on the effects of curcumin in osteoarthritic patients revealed that curcumin could reduce pain and improve the functionality of the patients (Table 1). These effects could be achieved as early as 4 weeks. Its effects were comparable to standard pharmacological agents, such as NSAIDs and glucosamine. These effects could be contributed to its antioxidant and anti-inflammatory activities. These mechanisms will be elaborated further in the following section.

\section{Antiarthritic mechanism of curcumin}

The mechanistic studies on the antiosteoarthritic properties of curcumin were performed on primary chondrocytes isolated from human, ${ }^{56-61}$ bovine, ${ }^{56}$ canine,${ }^{62}$ equine, ${ }^{63}$ or leporine ${ }^{64}$ cartilage, or immortal cell line. ${ }^{65}$ Cultures of chondrocyte monolayer and explant have been attempted. Two studies also examined the effect of curcumin on synoviocytes, ${ }^{66,67}$ another important constituent of the joint. Another study assessed the effects of mesenchymal stem cells that give rise to chondrocytes. ${ }^{62}$ Most studies induced a state of inflammation in the culture using IL-1 $\beta$. The main mechanisms studied to explain the antiarthritic actions of curcumin included chondrocyte regeneration and apoptosis, inflammation, and oxidative stress.

The vicious cycle of inflammation transpiring in the microenvironment of joint space favors the destruction of chondrocytes and suppresses their regeneration, thus producing a net cartilage loss. It would be intriguing to determine whether curcumin can reverse this process by enhancing the proliferation of chondrocytes and inhibiting their apoptosis. Studies showed that curcumin at low concentration $(<25 \mu \mathrm{M})$ was not toxic to equine chondrocytes monolayer or explant culture. ${ }^{63}$ Curcumin at higher concentration $(5 \mathrm{mM})$ did not promote or inhibit proliferation of immortalized C-28/I2 chondrocytes. ${ }^{65}$ Chondrogenesis was not promoted in canine mesenchymal stem cells cultured with curcumin. ${ }^{62}$ However, curcumin prevented IL-1 $\beta$ induced suppression of chondrogenesis, indicated by elevated markers of chondrocytic differentiation markers, such as chondroitin sulfate proteoglycans, collagen type II and $\beta 1$-integrin, and Src-homology collagen, compared to untreated cells. ${ }^{62}$ Curcumin also prevented apoptosis of canine chondrocytes induced by IL-1 $\beta$, indicated by reduced capases- 3 expression. ${ }^{62}$ Similar results were obtained in human primary chondrocytes cultures, whereby cellular features of apoptosis, such as development of multiple vacuoles, swelling of rough endoplasmic reticulum, clustering of swollen mitochondria and other organelles, condensation of heterochromatin in the nuclei, development of autophagic cytoplasmic vacuoles, disappearance of microvilli-like processes, and swelling of human primary chondrocytes, were reversed by treatment with curcumin for 15-30 minutes. ${ }^{61}$ Curcumin reversed apoptosis of chondrocytes induced by IL- $1 \beta$ via increasing the expression at antiapoptotic protein, such as Bcl-2, Bcl-XL, and TNF receptor-associated factor 1, and suppressing caspase-3. ${ }^{57}$ Further studies showed that mitogen-activated protein kinases pathway was implicated in the antiapoptotic action of curcumin. Pretreatment with $10 \mu \mathrm{M}$ curcumin prevented apoptosis in human chondrocytes caused by U0126, an inhibitor of mitogen-activated protein kinases. It also suppressed the activation of extracellular signal-regulated kinases 1 and 2, thereby preventing the activation of caspase- 3 and degradation of integrin. ${ }^{60}$ Interestingly, a study by Lev-Ari et al showed that a combination treatment of curcumin and celecoxib induced apoptosis of human synovial adherent cells, a source of inflammatory cytokines in the joint space. ${ }^{66}$

Inflammation within the joint space is aggravated by proteoglycans and MMPs or a disintegrin and metalloproteinase with thrombospondin motifs (ADAMTS) released from chondrocytes. Curcumin was able to suppress the expression of MMP-3 and MMP-9, COX-2 (enzyme responsible for synthesis of prostaglandin E-2 [PGE-2]), and PGE-2 (mediator of inflammation) in human primary chondrocytes induced by IL- $1 \beta$ or TNF $\alpha .^{58,59,67,68}$ Comblain et al demonstrated that curcuminoids decreased the gene expressions of MMP-3, ADAMTS4, ADAMTS5, COX-2, PGE-2, and IL-6. ${ }^{56}$ They also found that the combination of curcuminoids, hydrolyzed collagen, and green tea extract were more effective in 


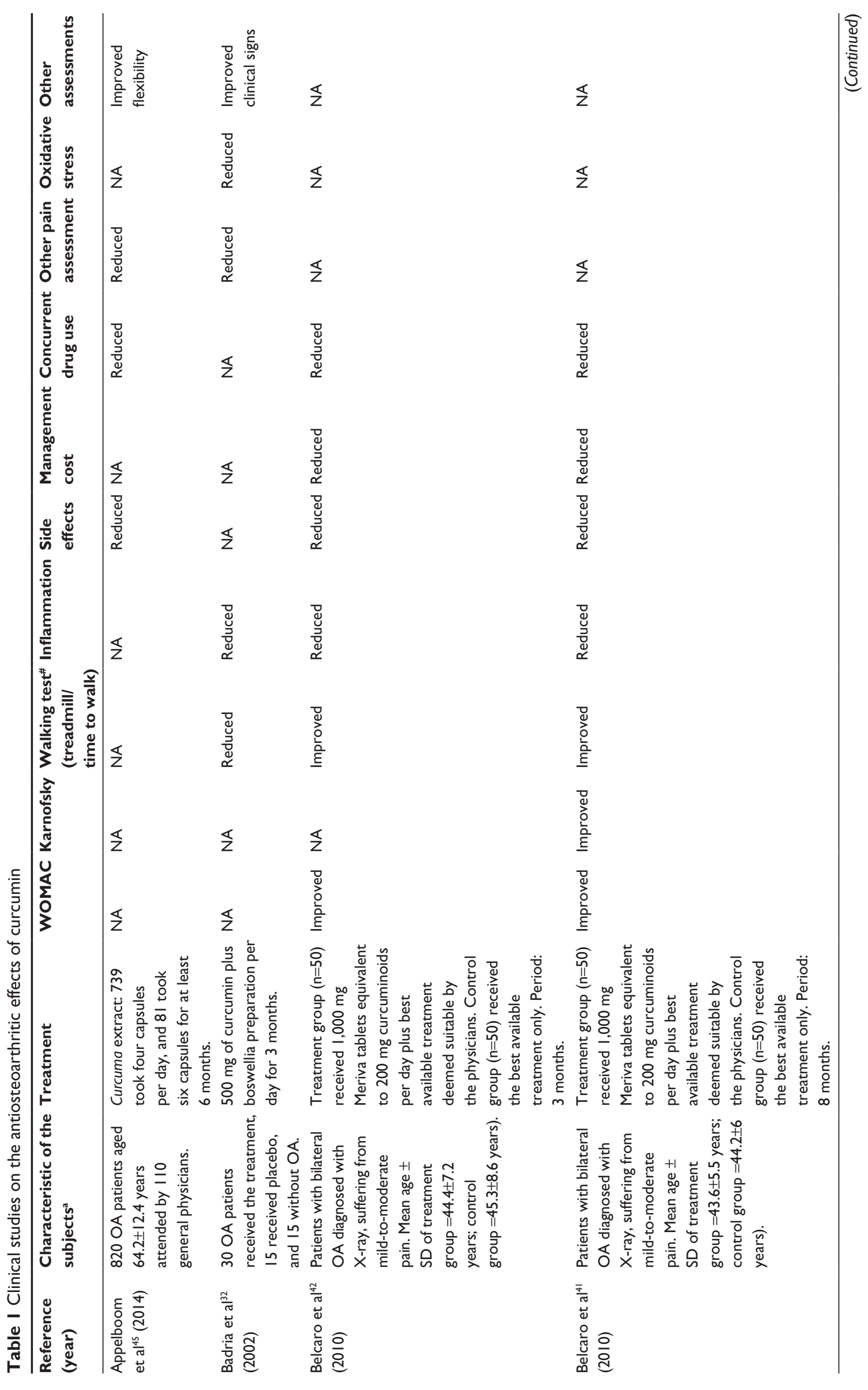




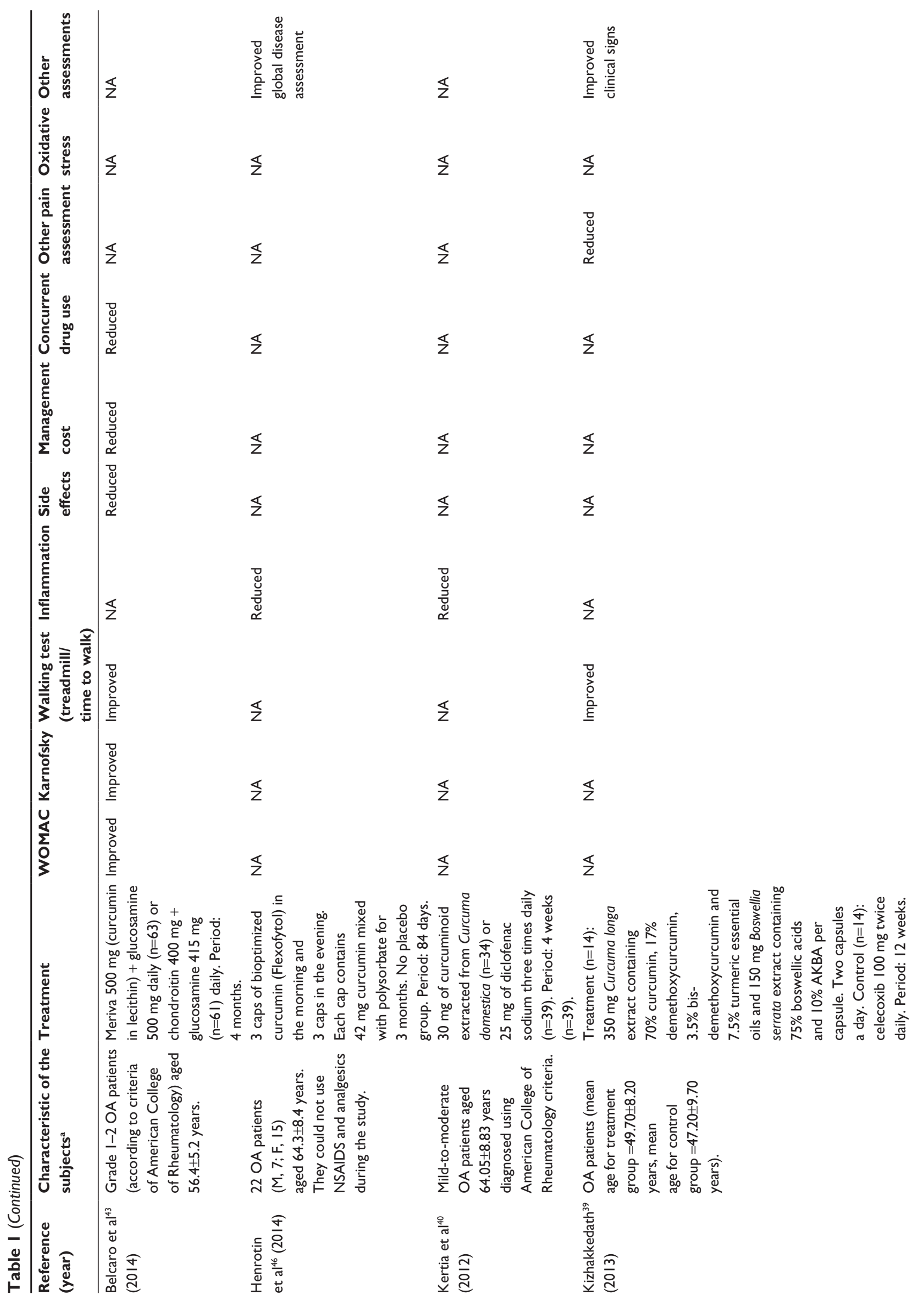




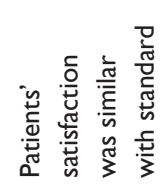

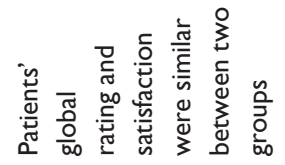

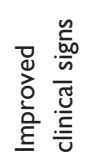

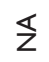

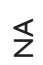

$\overleftarrow{z}$

$\stackrel{s}{z}$

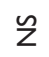

$\S$

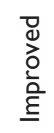

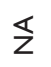

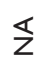

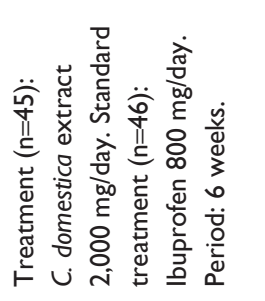

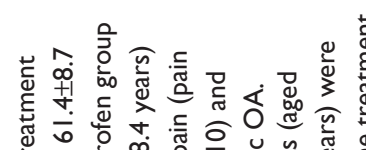

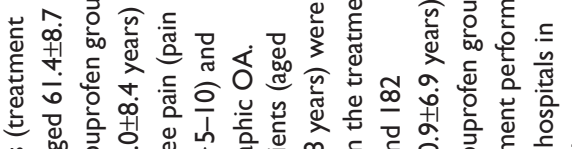

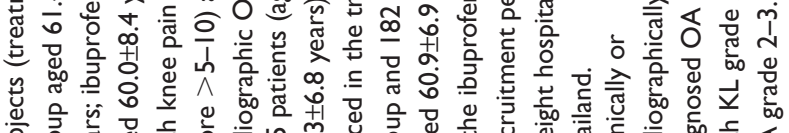

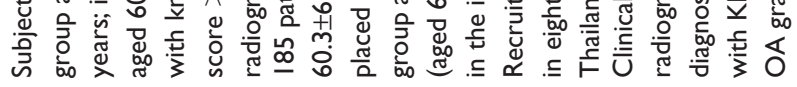

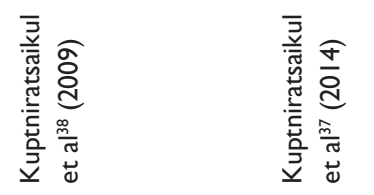

$\stackrel{s}{z}$

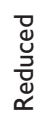

$\underset{\mathscr{\Xi}}{\stackrel{\Xi}{\mathscr{g}}}$

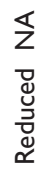

$\stackrel{s}{z}$

s

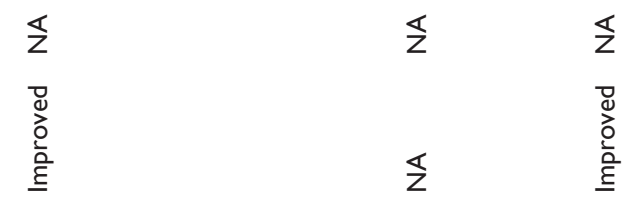

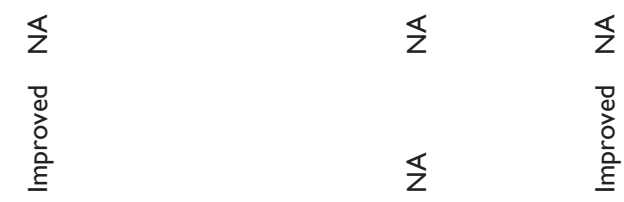

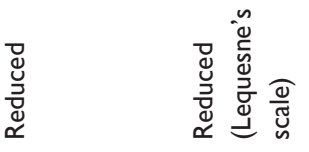

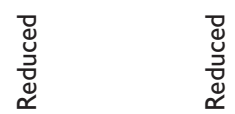

$\overleftarrow{z} \quad \mathbb{z}$

$\varangle \quad \varangle$

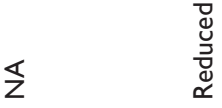

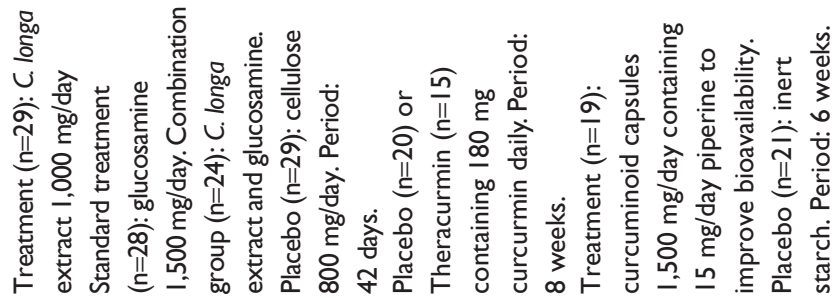

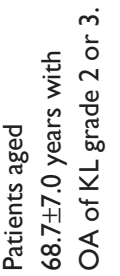

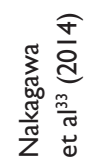

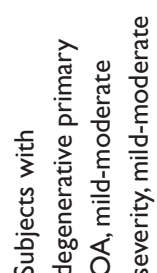

离 


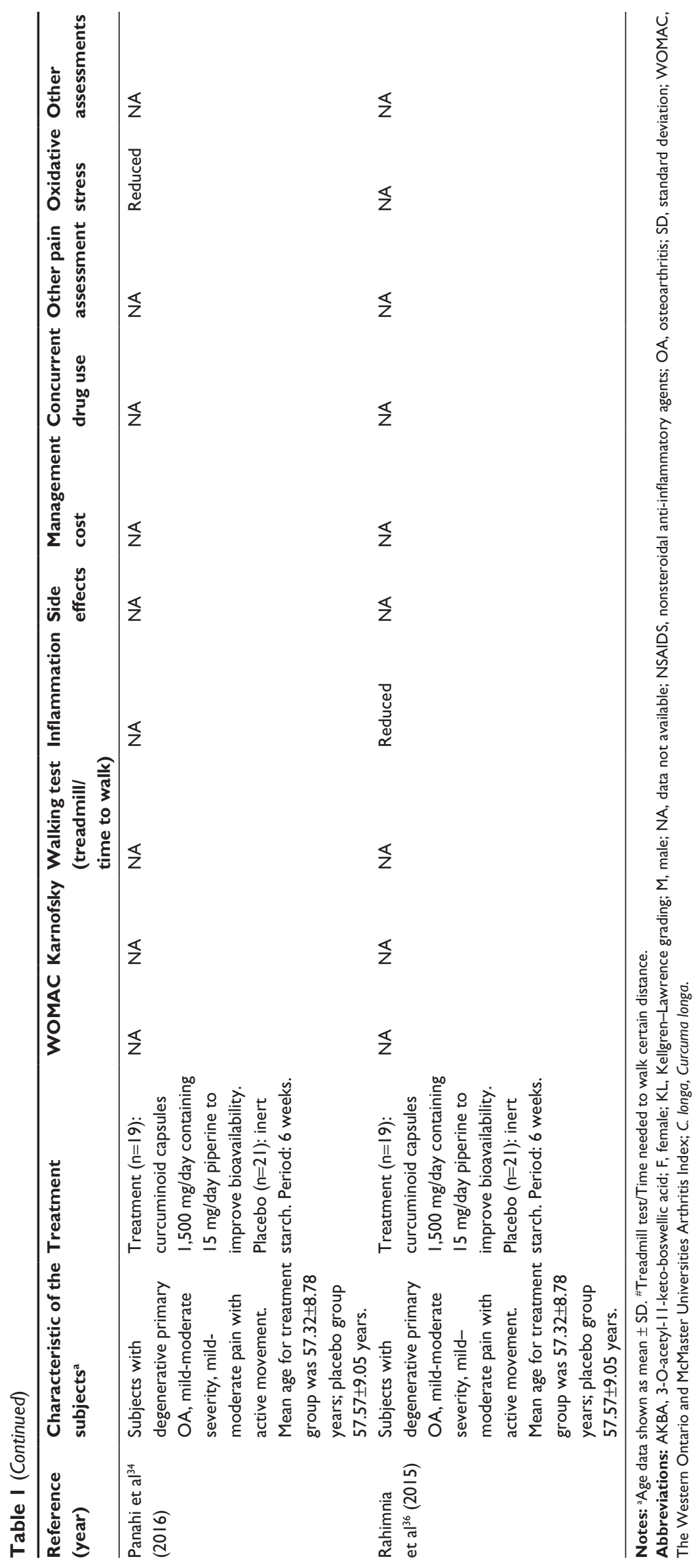


suppressing these genes than curcuminoids alone. ${ }^{56}$ Currently, curcumin reduced the level of MMP-3 and aggrecans released into the media by the bovine chondrocytes. ${ }^{56}$ This was in contrast with the findings of Mathy-Hartert et al, whereby production of aggrecans by chondrocytes was not affected by curcumin. ${ }^{68}$ Furthermore, they showed that curcumin was able to inhibit proteoglycan degradation in human chondrocytes in basal state but not in IL-1 $\beta$ stimulated model. ${ }^{68}$ Besides, expression of tissue inhibitor of metalloproteinase-1 was not affected by curcumin treatment in their study. ${ }^{68} \mathrm{On}$ the other hand, combination treatment of curcumin and celecoxib was able to suppress PGE-2 production in synoviocyte culture without affecting the protein expression of COX. ${ }^{66}$ However, curcumin increased the expression of MMP-3 and ADAMTS4 in immortalized C-28/I2 chondrocytes, indicating that curcumin might act distinctly in different in vitro chondrocyte models. ${ }^{65}$

Ultimately, the cartilage-protective and anti-inflammatory action of curcumin could be derived from its actions on the $\mathrm{NF}-\kappa \mathrm{B}$ system, which mediates the cellular inflammatory response (Figure 2). The NF- $\mathrm{KB}$ system is initiated by the activation of IKK. It phosphorylates IKB $\alpha$ and causes it

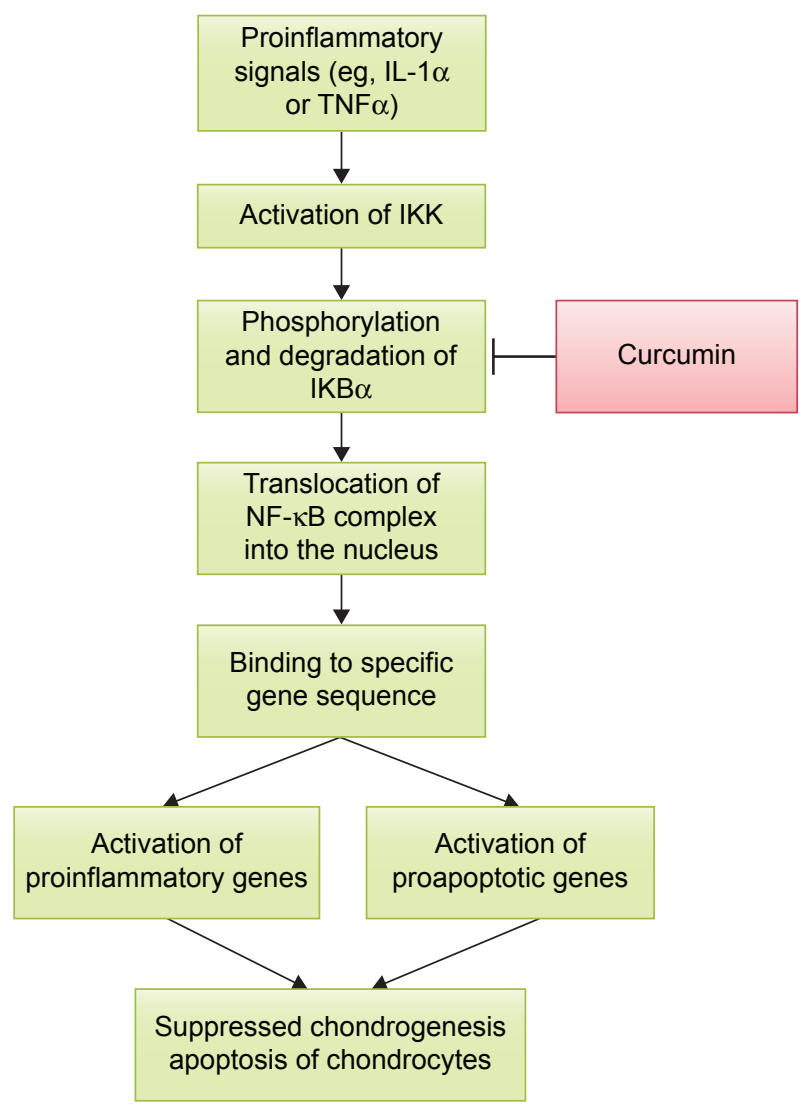

Figure 2 The action of curcumin on NF- $\kappa B$ pathway.

Abbreviations: IKK, IKB kinase; IL- $\mid \alpha$, interleukin I alpha; NF- $\kappa B$, nuclear factor kappa-light-chain-enhancer of activated B cells; TNF $\alpha$, tumor necrosis factor alpha. to degrade by ubiquitination. The freed NF- $\mathrm{kB}$ complex (a dimer consisting of p50 and p65) then enters the nucleus and switches on genes responsible for inflammation. ${ }^{69}$ In vitro studies strongly suggested that curcumin was able to prevent the phosphorylation and translocation of p65 into the nucleus of chondrocytes. ${ }^{57-59,62}$ Shakibaei et al showed that curcumin inhibited the phosphorylation of IKB $\alpha$ and AKT induced by IL-1 $\beta$ in human primary chondrocytes. ${ }^{59}$ However, further studies showed that expression and degradation of IKB $\alpha$ in chondrocytes were not affected by curcumin. ${ }^{57,59}$ Curcumin also did not alter the activation of IKK in chondrocytes. ${ }^{57}$

Limited studies regarding the effects of curcumin on oxidative status of chondrocytes were available. Yang et al demonstrated that treatment with curcumin was able to suppress the level of reactive oxygen species and lipid peroxidation products in primary leporine chondrocytes treated with advanced glycation product. This was attributed to the ability of curcumin in increasing the activity of catalase and superoxide dismutase. ${ }^{64}$

\section{Potential research area}

Despite the many clinical trials on osteoarthritis and curcumin, only a multiple-center study was found in literature. Researchers should consider more well-planned randomized controlled trials with a larger sample size and a longer treatment period on curcumin to justify its use in treating osteoarthritis. The efficacy of curcumin in treating older patients with more severe forms of osteoarthritis should also be validated. It will help to delay the need for total knee arthroplasty, which is a costly procedure with some undesirable side effects. Knowledge on the drug delivery system should be harnessed to improve the bioavailability of curcumin, which in its crude form, is poorly absorbed and quickly excreted by the body. This will also help to develop curcumin from a traditional supplement to a patterned pharmaceutical, thus improving its commercial value and marketability.

\section{Conclusion}

Osteoarthritis is a significant medical condition for the aging population worldwide. Curcumin, a NF-אB suppressor, demonstrates potential as a treatment agent for osteoarthritis, a disease with an underlying inflammatory cause. Its efficacy in reducing pain, physical function, and quality of life among osteoarthritic patients has been demonstrated in many clinical trials. The effects of curcumin on osteoarthritis can be attributed to its ability to prevent apoptosis of chondrocytes due to inflammation predominantly, and oxidative stress to a lesser extent. Randomized controlled trials involving larger sample size and longer treatment, proper selection of patients, 
and improved formulation could better justify the use of curcumin as an alternative treatment for osteoarthritis.

\section{Acknowledgments}

The author thanks Universiti Kebangsaan Malaysia for funding this study via FF-2016-119 and GGPM-2015-036 and Mr Mark Lee Wun Fui from the School of Chemical Sciences and Food Technology, Faculty of Science and Technology, Universiti Kebangsaan Malaysia for providing the chemical structures of curcumin, demethoxycurcumin, and bisdemethoxycurcumin.

\section{Disclosure}

The author reports no conflicts of interest in this work.

\section{References}

1. Kraus VB, Blanco FJ, Englund M, Karsdal MA, Lohmander LS. Call for standardized definitions of osteoarthritis and risk stratification for clinical trials and clinical use. Osteoarthritis Cartilage. 2015;23(8): 1233-1241.

2. Altman R, Asch E, Bloch D, et al. Development of criteria for the classification and reporting of osteoarthritis. Classification of osteoarthritis of the knee. Diagnostic and therapeutic criteria committee of the American Rheumatism Association. Arthritis Rheum. 1986;29(8):1039-1049.

3. Centers for Disease Control and Prevention. Osteoarthritis (OA); 2015. Available from: http://www.cdc.gov/arthritis/basics/osteoarthritis.htm. Accessed June 18, 2016.

4. Cross M, Smith E, Hoy D, et al. The global burden of hip and knee osteoarthritis: estimates from the global burden of disease 2010 study. Ann Rheum Dis. 2014;73(7):1323-1330.

5. Dillon CF, Rasch EK, Gu Q, Hirsch R. Prevalence of knee osteoarthritis in the United States: arthritis data from the Third National Health and Nutrition Examination Survey 1991-1994. J Rheumatol. 2006;33(11): 2271-2279.

6. Nuesch E, Dieppe P, Reichenbach S, Williams S, Iff S, Juni P. All cause and disease specific mortality in patients with knee or hip osteoarthritis: population based cohort study. BMJ. 2011;342:d1165.

7. Vos T, Flaxman AD, Naghavi M, et al. Years lived with disability (YLDs) for 1160 sequelae of 289 diseases and injuries 1990-2010: a systematic analysis for the Global Burden of Disease Study 2010. Lancet. 2012;380(9859):2163-2196.

8. Ruiz D Jr, Koenig L, Dall TM, et al. The direct and indirect costs to society of treatment for end-stage knee osteoarthritis. J Bone Joint Surg Am. 2013;95(16):1473-1480.

9. Benito MJ, Veale DJ, FitzGerald O, van den Berg WB, Bresnihan B. Synovial tissue inflammation in early and late osteoarthritis. Ann Rheum Dis. 2005;64(9):1263-1267.

10. Sohn DH, Sokolove J, Sharpe O, et al. Plasma proteins present in osteoarthritic synovial fluid can stimulate cytokine production via Toll-like receptor 4. Arthritis Res Ther. 2012;14(1):R7.

11. Sokolove J, Lepus CM. Role of inflammation in the pathogenesis of osteoarthritis: latest findings and interpretations. Ther Adv Musculoskelet Dis. 2013;5(2):77-94.

12. Blanco FJ, Rego I, Ruiz-Romero C. The role of mitochondria in osteoarthritis. Nat Rev Rheumatol. 2011;7(3):161-169.

13. Hochberg MC, Altman RD, April KT, et al. American College of Rheumatology 2012 recommendations for the use of nonpharmacologic and pharmacologic therapies in osteoarthritis of the hand, hip, and knee. Arthritis Care Res (Hoboken). 2012;64(4):465-474.

14. Nelson AE, Allen KD, Golightly YM, Goode AP, Jordan JM. A systematic review of recommendations and guidelines for the management of osteoarthritis: the chronic osteoarthritis management initiative of the U.S. bone and joint initiative. Semin Arthritis Rheum. 2014;43(6):701-712.
15. Zhang W, Moskowitz RW, Nuki G, et al. OARSI recommendations for the management of hip and knee osteoarthritis, part II: OARSI evidence-based, expert consensus guidelines. Osteoarthritis Cartilage. 2008;16(2):137-162.

16. Zhang W, Nuki G, Moskowitz RW, et al. OARSI recommendations for the management of hip and knee osteoarthritis: part III: changes in evidence following systematic cumulative update of research published through January 2009. Osteoarthritis Cartilage. 2010;18(4): 476-499.

17. Zhang W, Moskowitz RW, Nuki G, et al. OARSI recommendations for the management of hip and knee osteoarthritis, part I: critical appraisal of existing treatment guidelines and systematic review of current research evidence. Osteoarthritis Cartilage. 2007;15(9):981-1000.

18. Varas-Lorenzo C, Riera-Guardia N, Calingaert B, et al. Myocardial infarction and individual nonsteroidal anti-inflammatory drugs metaanalysis of observational studies. Pharmacoepidemiol Drug Saf. 2013; 22(6):559-570.

19. Skou ST, Roos EM, Laursen MB, et al. A randomized, controlled trial of total knee replacement. N Engl J Med. 2015;373(17):1597-1606.

20. Prasad S, Gupta SC, Tyagi AK, Aggarwal BB. Curcumin, a component of golden spice: from bedside to bench and back. Biotechnol Adv. 2014; 32(6):1053-1064

21. Pari L, Tewas D, Eckel J. Role of curcumin in health and disease. Arch Physiol Biochem. 2008;114(2):127-149.

22. Buhrmann C, Mobasheri A, Busch F, et al. Curcumin modulates nuclear factor kappaB (NF-kappaB)-mediated inflammation in human tenocytes in vitro: role of the phosphatidylinositol 3-kinase/Akt pathway. $J$ Biol Chem. 2011;286(32):28556-28566.

23. Fu XY, Yang MF, Cao MZ, et al. Strategy to suppress oxidative damage-induced neurotoxicity in PC12 cells by curcumin: the role of ROS-mediated DNA damage and the MAPK and AKT pathways. Mol Neurobiol. 2016;53(1):369-378.

24. Wu J, Li Q, Wang X, et al. Neuroprotection by curcumin in ischemic brain injury involves the Akt/Nrf2 pathway. PLoS One. 2013;8(3): e59843.

25. Cui Q, Li X, Zhu H. Curcumin ameliorates dopaminergic neuronal oxidative damage via activation of the Akt/Nrf2 pathway. Mol Med Rep. 2016;13(2):1381-1388.

26. Ak T, Gulcin I. Antioxidant and radical scavenging properties of curcumin. Chem Biol Interact. 2008;174(1):27-37.

27. Panahi Y, Sahebkar A, Parvin S, Saadat A. A randomized controlled trial on the anti-inflammatory effects of curcumin in patients with chronic sulphur mustard-induced cutaneous complications. Ann Clin Biochem. 2012;49(Pt 6):580-588.

28. Panahi Y, Saadat A, Beiraghdar F, Sahebkar A. Adjuvant therapy with bioavailability-boosted curcuminoids suppresses systemic inflammation and improves quality of life in patients with solid tumors: a randomized double-blind placebo-controlled trial. Phytother Res. 2014;28(10):1461-1467.

29. Singh AK, Vinayak M. Curcumin attenuates CFA induced thermal hyperalgesia by modulation of antioxidant enzymes and down regulation of TNF-alpha, IL-1beta and IL-6. Neurochem Res. 2015;40(3):463-472.

30. $\mathrm{Xu} \mathrm{D}, \mathrm{Hu} \mathrm{L}, \mathrm{Su} \mathrm{C}$, et al. Tetrachloro-p-benzoquinone induces hepatic oxidative damage and inflammatory response, but not apoptosis in mouse: the prevention of curcumin. Toxicol Appl Pharmacol. 2014; 280(2):305-313.

31. Moon DO, Kim MO, Choi YH, Park YM, Kim GY. Curcumin attenuates inflammatory response in IL-1beta-induced human synovial fibroblasts and collagen-induced arthritis in mouse model. Int Immunopharmacol. 2010;10(5):605-610.

32. Badria FA, El-Farahaty T, Shabana AA, Hawas SA, El-Batoty MF. Boswellia-curcumin preparation for treating knee osteoarthritis: a clinical evaluation. Altern Complement Ther. 2002;8(6):341-348.

33. Nakagawa Y, Mukai S, Yamada S, et al. Short-term effects of highlybioavailable curcumin for treating knee osteoarthritis: a randomized, double-blind, placebo-controlled prospective study. J Orthop Sci. 2014; 19(6):933-939. 
34. Panahi Y, Alishiri GH, Parvin S, Sahebkar A. Mitigation of systemic oxidative stress by curcuminoids in osteoarthritis: results of a randomized controlled trial. J Diet Suppl. 2016;13(2):209-220.

35. Panahi Y, Rahimnia AR, Sharafi M, Alishiri G, Saburi A, Sahebkar A. Curcuminoid treatment for knee osteoarthritis: a randomized double-blind placebo-controlled trial. Phytother Res. 2014;28(11):1625-1631.

36. Rahimnia AR, Panahi Y, Alishiri G, Sharafi M, Sahebkar A. Impact of supplementation with curcuminoids on systemic inflammation in patients with knee osteoarthritis: findings from a randomized double-blind placebo-controlled trial. Drug Res (Stuttg). 2015;65(10):521-525.

37. Kuptniratsaikul V, Dajpratham P, Taechaarpornkul W, et al. Efficacy and safety of Curcuma domestica extracts compared with ibuprofen in patients with knee osteoarthritis: a multicenter study. Clin Interv Aging. 2014;9:451-458

38. Kuptniratsaikul V, Thanakhumtorn S, Chinswangwatanakul P, Wattanamongkonsil L, Thamlikitkul V. Efficacy and safety of Curcuma domestica extracts in patients with knee osteoarthritis. J Altern Complement Med. 2009;15(8):891-897.

39. Kizhakkedath R. Clinical evaluation of a formulation containing Curcuma longa and Boswellia serrata extracts in the management of knee osteoarthritis. Mol Med Rep. 2013;8(5):1542-1548.

40. Kertia N, Asdie AH, Rochmah W, Marsetyawan. Ability of curcuminoid compared to diclofenac sodium in reducing the secretion of cycloxygenase- 2 enzyme by synovial fluid's monocytes of patients with osteoarthritis. Acta Med Indones. 2012;44(2):105-113.

41. Belcaro G, Cesarone MR, Dugall M, et al. Efficacy and safety of Meriva(R), a curcumin-phosphatidylcholine complex, during extended administration in osteoarthritis patients. Altern Med Rev. 2010;15(4): 337-344

42. Belcaro G, Cesarone MR, Dugall M, et al. Product-evaluation registry of Meriva(R), a curcumin-phosphatidylcholine complex, for the complementary management of osteoarthritis. Panminerva Med. 2010; 52(2 Suppl 1):55-62.

43. Belcaro G, Dugall M, Luzzi R, et al. Meriva(R)+Glucosamine versus Chondroitin + Glucosamine in patients with knee osteoarthritis: an observational study. Eur Rev Med Pharmacol Sci. 2014;18(24):3959-3963.

44. Madhu K, Chanda K, Saji MJ. Safety and efficacy of Curcuma longa extract in the treatment of painful knee osteoarthritis: a randomized placebocontrolled trial. Inflammopharmacology. 2013;21(2):129-136.

45. Appelboom T, Maes N, Albert A. A new curcuma extract (flexofytol(R)) in osteoarthritis: results from a belgian real-life experience. Open Rheumatol J. 2014;8:77-81.

46. Henrotin Y, Gharbi M, Dierckxsens Y, et al. Decrease of a specific biomarker of collagen degradation in osteoarthritis, Coll2-1, by treatment with highly bioavailable curcumin during an exploratory clinical trial. BMC Complement Altern Med. 2014;14:159.

47. Kulkarni RR, Patki PS, Jog VP, Gandage SG, Patwardhan B. Treatment of osteoarthritis with a herbomineral formulation: a doubleblind, placebo-controlled, cross-over study. J Ethnopharmacol. 1991 33(1-2):91-95.

48. Yang KY, Lin LC, Tseng TY, Wang SC, Tsai TH. Oral bioavailability of curcumin in rat and the herbal analysis from Curcuma longa by LC-MS/MS. J Chromatogr B Analyt Technol Biomed Life Sci. 2007; 853(1-2):183-189.

49. Liu W, Zhai Y, Heng X, et al. Oral bioavailability of curcumin: problems and advancements. J Drug Target. 2016;24(8):694-702.

50. Marczylo TH, Verschoyle RD, Cooke DN, Morazzoni P, Steward WP, Gescher AJ. Comparison of systemic availability of curcumin with that of curcumin formulated with phosphatidylcholine. Cancer Chemother Pharmacol. 2007;60(2):171-177.

51. Sunagawa Y, Hirano S, Katanasaka Y, et al. Colloidal submicron-particle curcumin exhibits high absorption efficiency-a double-blind, 3-way crossover study. J Nutr Sci Vitaminol (Tokyo). 2015;61(1):37-44.

52. Morimoto T, Sunagawa Y, Katanasaka Y, et al. Drinkable preparation of Theracurmin exhibits high absorption efficiency - a single-dose, double-blind, 4-way crossover study. Biol Pharm Bull. 2013;36(11): 1708-1714.
53. Kanai M, Otsuka Y, Otsuka K, et al. A phase I study investigating the safety and pharmacokinetics of highly bioavailable curcumin (Theracurmin) in cancer patients. Cancer Chemother Pharmacol. 2013;71(6): 1521-1530.

54. Reen RK, Jamwal DS, Taneja SC, et al. Impairment of UDP-glucose dehydrogenase and glucuronidation activities in liver and small intestine of rat and guinea pig in vitro by piperine. Biochem Pharmacol. 1993;46(2):229-238.

55. Shoba G, Joy D, Joseph T, Majeed M, Rajendran R, Srinivas PS. Influence of piperine on the pharmacokinetics of curcumin in animals and human volunteers. Planta Med. 1998;64(4):353-356.

56. Comblain F, Sanchez C, Lesponne I, Balligand M, Serisier S, Henrotin Y. Curcuminoids extract, hydrolyzed collagen and green tea extract synergically inhibit inflammatory and catabolic mediator's synthesis by normal bovine and osteoarthritic human chondrocytes in monolayer. PLoS One. 2015;10(3):e0121654.

57. Csaki C, Mobasheri A, Shakibaei M. Synergistic chondroprotective effects of curcumin and resveratrol in human articular chondrocytes: inhibition of IL-1beta-induced NF-kappaB-mediated inflammation and apoptosis. Arthritis Res Ther. 2009;11(6):R165.

58. Schulze-Tanzil G, Mobasheri A, Sendzik J, John T, Shakibaei M. Effects of curcumin (diferuloylmethane) on nuclear factor kappaB signaling in interleukin-1beta-stimulated chondrocytes. Ann N Y Acad Sci. 2004;1030:578-586.

59. Shakibaei M, John T, Schulze-Tanzil G, Lehmann I, Mobasheri A. Suppression of NF-kappaB activation by curcumin leads to inhibition of expression of cyclo-oxygenase- 2 and matrix metalloproteinase- 9 in human articular chondrocytes: implications for the treatment of osteoarthritis. Biochem Pharmacol. 2007;73(9):1434-1445.

60. Shakibaei M, Mobasheri A, Buhrmann C. Curcumin synergizes with resveratrol to stimulate the MAPK signaling pathway in human articular chondrocytes in vitro. Genes Nutr. 2011;6(2):171-179.

61. Shakibaei M, Schulze-Tanzil G, John T, Mobasheri A. Curcumin protects human chondrocytes from IL-11beta-induced inhibition of collagen type II and beta1-integrin expression and activation of caspase-3: an immunomorphological study. Ann Anat. 2005;187(5-6): 487-497.

62. Buhrmann C, Mobasheri A, Matis U, Shakibaei M. Curcumin mediated suppression of nuclear factor-kappaB promotes chondrogenic differentiation of mesenchymal stem cells in a high-density co-culture microenvironment. Arthritis Res Ther. 2010;12(4):R127.

63. Clutterbuck AL, Allaway D, Harris P, Mobasheri A. Curcumin reduces prostaglandin E2, matrix metalloproteinase-3 and proteoglycan release in the secretome of interleukin 1beta-treated articular cartilage. F1000Res. 2013;2:147.

64. Yang Q, Wu S, Mao X, Wang W, Tai H. Inhibition effect of curcumin on TNF-alpha and MMP-13 expression induced by advanced glycation end products in chondrocytes. Pharmacology. 2013;91(1-2):77-85.

65. Toegel S, Wu SQ, Piana C, et al. Comparison between chondroprotective effects of glucosamine, curcumin, and diacerein in IL-1betastimulated C-28/I2 chondrocytes. Osteoarthritis Cartilage. 2008; 16(10):1205-1212.

66. Lev-Ari S, Strier L, Kazanov D, et al. Curcumin synergistically potentiates the growth-inhibitory and pro-apoptotic effects of celecoxib in osteoarthritis synovial adherent cells. Rheumatology (Oxford). 2006; 45(2):171-177.

67. TenBroek EM, Yunker L, Nies MF, Bendele AM. Randomized controlled studies on the efficacy of antiarthritic agents in inhibiting cartilage degeneration and pain associated with progression of osteoarthritis in the rat. Arthritis Res Ther. 2016;18:24.

68. Mathy-Hartert M, Jacquemond-Collet I, Priem F, Sanchez C, Lambert C, Henrotin Y. Curcumin inhibits pro-inflammatory mediators and metalloproteinase-3 production by chondrocytes. Inflamm Res. 2009 ; 58(12):899-908.

69. van Delft MA, Huitema LF, Tas SW. The contribution of NF-kappaB signalling to immune regulation and tolerance. Eur J Clin Invest. 2015; 45(5):529-539. 


\section{Publish your work in this journal}

Drug Design, Development and Therapy is an international, peerreviewed open-access journal that spans the spectrum of drug design and development through to clinical applications. Clinical outcomes, patient safety, and programs for the development and effective, safe, and sustained use of medicines are the features of the journal, which

has also been accepted for indexing on PubMed Central. The manuscript management system is completely online and includes a very quick and fair peer-review system, which is all easy to use. Visit http://www.dovepress.com/testimonials.php to read real quotes from published authors.

Submit your manuscript here: http://www.dovepress.com/drug-design-development-and-therapy-journal 